\title{
AVALIAÇÃO DE ALGUNS PARÂMETROS FÍSICO-QUÍMICOS DA ÁGUA DE UMA PISCICULTURA COM SISTEMA DE CULTIVO EM TANQUES-REDE
}

Juliana Heloisa Pinê Américo ${ }^{1}$

Gláucio Doreide Cicigliano ${ }^{2}$

Sérgio Luís de Carvalho ${ }^{3}$

RESUMO: A piscicultura apesar de ser uma atividade econômica que depende da água, é considerada como fonte poluidora dos ambientes aquáticos, principalmente devido à introdução de substâncias que podem alterar alguns parâmetros da qualidade da água. O presente trabalho teve como objetivo avaliar a temperatura e os parâmetros químicos de $\mathrm{pH}$, oxigênio dissolvido, nitrogênio total e fósforo total da água de uma piscicultura com sistema de cultivo em tanques-rede na Bacia Hidrográfica do Rio São José dos Dourados, Ribeirão da Ponte Pensa no município de Santa Fé do Sul e verificar se estes parâmetros enquadram-se nos limites estabelecidos pelo CONAMA 357/05. A temperatura, o pH e a concentração de oxigênio dissolvido foram mensurados com auxílio de um termômetro, pHmetro e oxímetro respectivamente. Para determinação da concentração de nitrogênio total e fósforo total realizaram-se análises laboratoriais baseadas nos Métodos de Análises de Águas Potáveis e Residuárias através de digestão com persulfato e pelo método espectrofotométrico. A temperatura da água manteve-se pouco variável, os valores de $\mathrm{pH}$, oxigênio dissolvido e fósforo total enquadraram-se na legislação, no entanto em algumas amostras a concentração de nitrogênio foi superior ao padrão estabelecido no CONAMA 357/05. Os resultados sugerem que a produção em tanques-rede da área estudada é uma atividade viável, desde que haja monitoramento da qualidade da água e otimização no uso da ração para que a mesma não seja desperdiçada, contribuindo com a eutrofização do ambiente aquático.

\footnotetext{
${ }_{1}^{1}$ Doutoranda em Aquicultura, Centro de Aquicultura da UNESP. E-mail: americo.ju@gmail.com

${ }^{2}$ Mestre em Engenharia Civil, Faculdade de Engenharia de Ilha Solteira (UNESP). E-mail: gldrcc@hotmail.com

${ }^{3}$ Docente, Faculdade de Engenharia de Ilha Solteira (UNESP). E-mail: sergicar@bio.feis.unesp.br
} 
Palavras-chave: Nitrogênio. Fósforo. Tilápia.

\section{INTRODUÇÃO}

A aquicultura moderna, além de contemplar o lucro, deve considerar a preservação ambiental como parte integrante do seu processo de produção, de modo que suas técnicas otimizem a produção de organismos aquáticos e considerem os possíveis impactos do sistema de cultivo (VALENTI, 2000). O maior problema ambiental no cultivo de peixes está relacionado aos seus efluentes com grande potencial de poluição das águas naturais. Embora estes efluentes não apresentem altas concentrações de poluentes quando comparados aos efluentes de indústrias e municipais, às vezes podem conter concentrações de algumas variáveis limnológicas acima daquelas permitidas, tornando-se uma fonte de poluição (BOYD e SCHIMITTOU, 1999).

As características dos efluentes de piscicultura podem variar em função de espécie cultivada, da densidade dos organismos cultivados, do manejo alimentar e do nível de tecnologia empregado no cultivo (BOYD, 2003). Durante o processo de produção piscícola a maior preocupação são as substâncias dissolvidas e em suspensão presentes nos seus efluentes, cuja maioria é oriunda de metabólitos e sobras de ração (QUEIROZ, 1998). O acúmulo de resíduos orgânicos e metabólitos nos tanques e viveiros dos sistemas de cultivo de renovação de água intermitente são inevitáveis, sendo que o volume de fezes excretado diariamente pela população de peixes é uma das principais fontes de resíduos orgânicos em sistemas aquiculturas. A digestibilidade da matéria seca das rações varia entre 70 e $75 \%$. Isto significa que 25 a $30 \%$ do alimento fornecido entra nos sistemas aquaculturais como material fecal (KUBITZA, 1998).

Os maiores impactos causados pela piscicultura em tanques-rede dizem respeito ao aumento nas concentrações de fósforo, nitrogênio e matéria orgânica, tanto na água quanto no sedimento (GUO e LI, 2003). Segundo Folke e Kautsky (1992), 13\% do nitrogênio e $66 \%$ do fósforo aportado via ração sofre sedimentação, $25 \%$ do nitrogênio e $23 \%$ do fósforo são convertidos em massa (carne) e $62 \%$ de nitrogênio e $11 \%$ de fósforo ficam dissolvidos na água. 
Este incremento de matéria orgânica favorece o processo de eutrofização artificial que pode comprometer os sistemas de cultivo em decorrência da redução da concentração de oxigênio dissolvido e do aumento de nutrientes na água, que pode ocasionar blooms de algas e bactérias. Dessa forma, a redução da qualidade da água nos sistemas de criação pode afetar a qualidade do pescado, com consequente prejuízo aos produtores. Além dos efeitos sobre os sistemas de produção, o impacto ambiental é também uma preocupação, uma vez que estes sistemas podem deteriorar a qualidade das águas naturais (BACCARIN e CAMARGO, 2005).

O presente trabalho teve como objetivo avaliar a temperatura e os parâmetros químicos de $\mathrm{pH}$, oxigênio dissolvido, nitrogênio total e fósforo total da água de uma piscicultura com sistema de cultivo em tanques-rede na Bacia Hidrográfica do Rio São José dos Dourados, Ribeirão da Ponte Pensa no município de Santa Fé do Sul - SP, durante onze meses e verificar se os resultados encontrados enquadram-se nos padrões estabelecidos pelo CONAMA 357/05.

\section{MATERIAL E MÉTODOS}

\subsection{Caracterização da área de estudo}

O município de Santa Fé do Sul localiza-se no Noroeste do Estado de São Paulo com uma área de $208,3 \mathrm{Km}^{2}$, população de 27.693 habitantes, clima tropical com inverno seco e está inserido na Bacia Hidrográfica do Rio São José dos Dourados (IBGE, 2007) (Figura 1) 


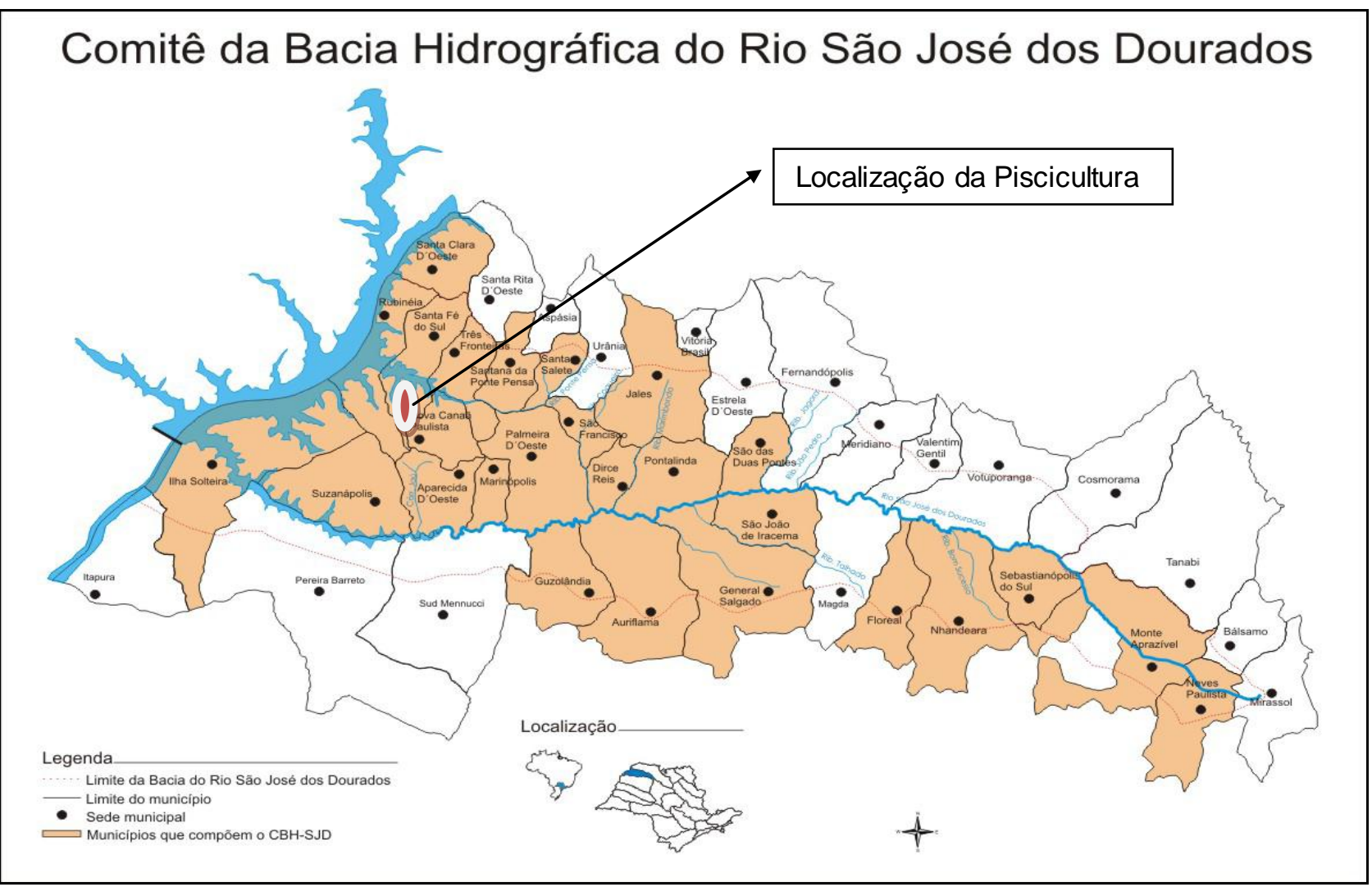

Figura 1. Localização da região do estudo (COMITÊ DA BACIA HIDROGRÁFICA DO RIO SÃO JOSÉ DOS DOURADOS, 2009).

O local definido para o estudo encontra-se entre as coordenadas: Datum Horizontal: SAD-69; Fuso UTM: 23; Coordenada UTM-E: -25382,001231445; Coordenada UTM-N: 7749068,55577131, à montante da Usina Hidrelétrica de Ilha Solteira que pertence a Companhia Energética de São Paulo (CESP), onde está instalada uma piscicultura no Ribeirão da Ponte Pensa, com sistema de cultivo constituído por 350 tanques-rede. Cada tanque-rede apresenta $18 \mathrm{~m}^{3}$ de volume útil, sendo que a área de cultivo da piscicultura é de 10 ha de espelho d’água.

A capacidade de produção estimada para esta piscicultura é de 100 toneladas de peso vivo de tilápia do Nilo (Oreochromis niloticus) ao mês com um consumo médio mensal de ração extrusada de 150 toneladas.

\subsection{Pontos de amostragem}


Três pontos de amostragem foram definidos na área de estudo, estes denominados Ponto 1 (ponto central de cultivo entre os tanques-rede), Ponto 2 ( $200 \mathrm{~m}$ da área de cultivo) e Ponto 3 (400 $\mathrm{m}$ da área de cultivo). As coletas de água foram realizadas mensalmente durante 11 meses a uma profundidade de $1,5 \mathrm{~m}$ no período da manhã. Vale salientar que no local onde a piscicultura está instalada (Ribeirão da Ponte Pensa) não ocorre um fluxo de deslocamento de água, por se tratar de um braço de rio sem influencia de nenhum afluente, motivo pelo quais as coletas foram realizadas somente lateralmente aos tanques.

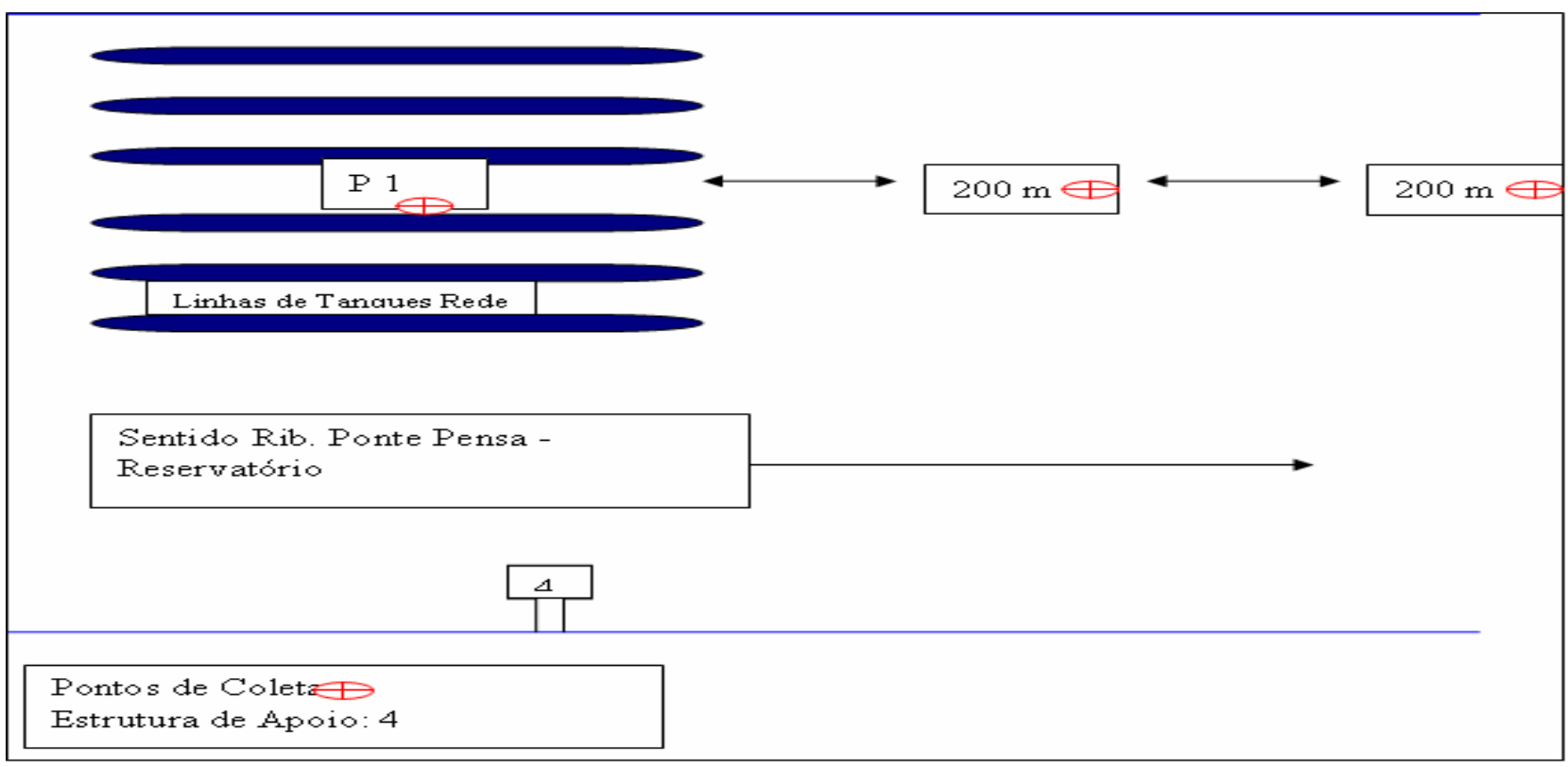

Figura 2. Localização dos pontos de coleta em relação à piscicultura no Ribeirão Ponte Pensa.

\subsection{Análises de campo}

Em cada ponto de amostragem foram mensurados a temperatura, $\mathrm{o} \mathrm{pH}$ e a concentração de oxigênio dissolvido com auxílio de um termômetro, pHmetro e oxímetro pertencentes ao Laboratório de Saneamento da Faculdade de Engenharia de Ilha Solteira (FEIS-UNESP).

\subsection{Análises de laboratório}




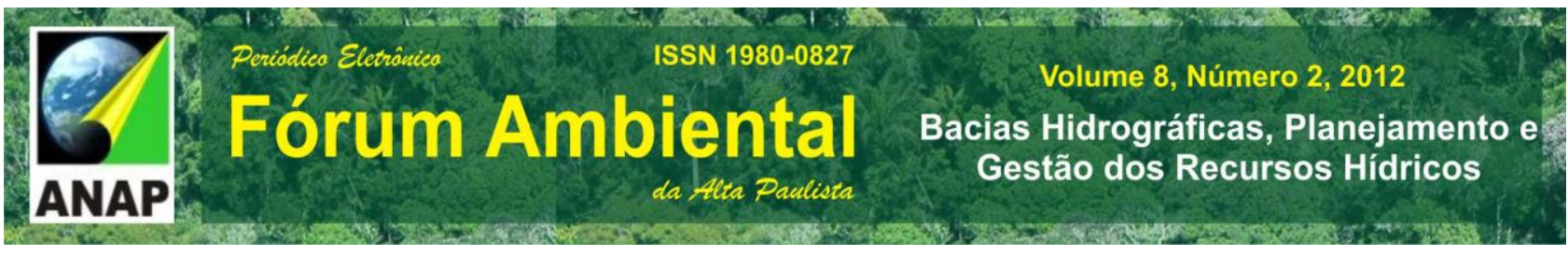

As análises para determinação da concentração de nitrogênio e fósforo dos pontos de amostragem foram realizadas no Laboratório de Saneamento da FEIS-UNESP com base nos Métodos para Análises de Águas Potáveis e Residuárias - Standard Methods for Examination of Water and Wastewater (APHA-AWWA-WPCF 1998).

Os parâmetros químicos de nitrogênio total e fósforo total foram determinados por digestão com persulfato e pelo método espectrofotométrico com utilização de um aparelho COD Reactor/Hach e espectrofotômetro Hach.

\section{RESULTADOS E DISCUSSÃO}

O Ribeirão da Ponte Pensa - SP não dispõe de uma classificação de suas águas. Assim, de acordo com a resolução CONAMA 357/05, em seu capítulo VI Artg. 42 "enquanto não aprovados os respectivos enquadramentos, as águas doces serão consideradas classe 2". Utilizou-se esta classificação com a finalidade de analisar as condições em que se encontra o corpo hídrico estudado.

A temperatura da água manteve-se com poucas variações nos diferentes pontos de coleta durante o estudo. A máxima temperatura mensurada ocorreu nos mês de janeiro $\left(30^{\circ} \mathrm{C}\right)$ e a mínima nos meses de junho e julho $\left(23^{\circ} \mathrm{C}\right)$ apresentando variações de temperatura conforme a caracterização do clima da bacia hidrográfica (Tabela 1).

As temperaturas registradas encontram-se no padrão considerado como conforto térmico para tilápias, pois segundo Kubitza (2000) as tilápias são peixes tropicais que apresentam conforto térmico entre 27 e $32^{\circ} \mathrm{C}$. Temperaturas acima de $32^{\circ} \mathrm{C}$ e abaixo de $26^{\circ} \mathrm{C}$ reduzem o apetite e o crescimento dos peixes. Abaixo de $20^{\circ} \mathrm{C}$ o apetite fica extremamente reduzido e aumentam os riscos de doenças. Com temperatura da água abaixo de $18^{\circ} \mathrm{C}$, o sistema imunológico das tilápias é suprimido.

Tabela 1. Temperatura da água, em ${ }^{\circ} \mathrm{C}$, nos pontos de amostragem da piscicultura no Córrego Ribeirão Ponte Pensa, Santa Fé do Sul -SP, no período de março de 2008 à fevereiro de 2009

\begin{tabular}{cccccccccccc}
\hline & \multicolumn{10}{c}{ Meses } \\
\cline { 2 - 5 } $\begin{array}{c}\text { Pontos } \\
\text { amostrais }\end{array}$ & Mar & Abr & Mai & Jun & Jul & Ago & Set & Out & Nov & Jan & Fev \\
\hline
\end{tabular}




$\begin{array}{llllllllllll}\text { P1 } & 28 & 28 & 25 & 23 & 23 & 24 & 27 & 29 & 29 & 30 & 29 \\ \text { P2 } & 28 & 28 & 25 & 23 & 23 & 24 & 27 & 29 & 29 & 30 & 29 \\ \text { P3 } & 28 & 28 & 25 & 23 & 23 & 24 & 27 & 29 & 29 & 30 & 29\end{array}$

Em relação ao pH, o maior valor $(7,77)$ foi registrado no mês de novembro de 2008 no Ponto 2 e o menor valor $(6,27)$ foi registrado no Ponto 1 no mês de março de 2008 (Tabela 2). Os valores de $\mathrm{pH}$ encontrados enquadram-se no padrão estabelecido pelo CONAMA 357/05 para corpos d'água de classe 2, cuja faixa estabelecida varia de 6,00 a 9,00 .

Tabela 2. Valores de pH da água nos pontos de amostragem da piscicultura no Córrego Ribeirão Ponte Pensa, Santa Fé do Sul -SP, no período de março de 2008 à fevereiro de 2009

\begin{tabular}{ccccccccccccc}
\hline \multirow{2}{*}{$\begin{array}{c}\text { Pontos } \\
\text { amostrais }\end{array}$} & Mar & Abr & Mai & Jun & Jul & Ago & Set & Out & Nov & Jan & Fev \\
\hline P1 & 6,27 & 6,28 & 6,76 & 6,79 & 6,91 & 6,74 & 6,62 & 7,07 & 6,5 & 6,95 & 7,04 \\
P2 & 6,31 & 6,49 & 7,03 & 7,05 & 6,99 & 6,89 & 6,71 & 7,32 & 7,77 & 7,66 & 6,81 \\
P3 & 6,3 & 6,3 & 6,43 & 7,08 & 6,98 & 6,88 & 6,79 & 7,56 & 7,01 & 7,62 & 7,19 \\
\hline
\end{tabular}

Os pontos letais de acidez e alcalinidade são de $\mathrm{pH} 4$ e pH 11, sendo a faixa de 6,5 a 9,0 a mais adequada para a produção de peixes. Tilápias apresentam baixa sobrevivência quando em águas com pH abaixo de 4,0 (BOYD, 2003).

A maior concentração de oxigênio dissolvido na água $\left(11 \mathrm{mg} \cdot \mathrm{L}^{-1}\right)$ foi registrada no mês de fevereiro de 2009 no ponto 3 localizado à 400m da piscicultura e a menor concentração $\left(5,38 \mathrm{mg} \cdot \mathrm{L}^{-1}\right)$ foi encontrada em abril de 2008 no Ponto 1 (Tabela 3), enquadrando-se no padrão estabelecido pelo CONAMA 357/05 para corpos d'água de classe 2, onde a concentração de oxigênio dissolvido em qualquer amostra de água não deve ser inferior a $5 \mathrm{mg} \cdot \mathrm{L}^{-1}$.

Tabela 3. Concentração de oxigênio dissolvido na água, $\mathrm{mg} \cdot \mathrm{L}^{-1}$, nos pontos de amostragem da piscicultura no Córrego Ribeirão Ponte Pensa, Santa Fé do Sul - SP, no período de março de 2008 à fevereiro de 2009

\section{Meses}




\section{Pontos}

2008

2009

amostrais Mar Abr Mai Jun Jul Ago Set Out Nov Jan Fev

$\begin{array}{lccccccccccc}\text { P1 } & 5,99 & 5,38 & 6,02 & 5,62 & 7,43 & 7,5 & 7,5 & 7,08 & 6,13 & 9,0 & 6,84 \\ \text { P2 } & 6,75 & 6,35 & 7,22 & 7,63 & 9,74 & 7,9 & 8,19 & 8,15 & 7,68 & 9,2 & 9,91 \\ \text { P3 } & 6,8 & 6,4 & 7,23 & 7,83 & 7,98 & 7,7 & 8,5 & 7,94 & 8,02 & 9,37 & 11\end{array}$

Para piscicultura, a concentração mínima de oxigênio dissolvido que deve ser mantida é de $4 \mathrm{mg} \cdot \mathrm{L}^{-1}$, quando esta concentração chega a valores abaixo de $2 \mathrm{mg} \cdot \mathrm{L}^{-1}$ pode ocorrer excessivo estresse e mortalidade de peixes (KUBITZA, 2000). Entretanto, a tilápia é um peixe que tolera baixas concentrações de oxigênio dissolvido e segundo Becker e Fishelsonl (1986) a concentração crítica para híbridos de Oreochromis niloticus x O. mossambicus é de 0,7 a $1,6 \mathrm{mg} \cdot \mathrm{L}^{-1}$, ou seja, 10 a $20 \%$ de saturação a $26-35^{\circ} \mathrm{C}$ de temperatura da água. Quando a concentração de oxigênio dissolvido atinge 45 a $50 \%$ de saturação (aproximadamente 3 a $3,5 \mathrm{mg} / \mathrm{L}$ a $28-30^{\circ} \mathrm{C}$ ), a tilápia começa a reduzir sua atividade e, portanto, o consumo de oxigênio (ROSS e ROSS, 1983).

As concentrações de nutrientes como nitrogênio e fósforo em aquicultura são parâmetros que despertam maior preocupação, pois geralmente estes nutrientes são provindos de metabólitos de peixes e sobras de ração. As maiores concentrações de nitrogênio total foram detectadas no ponto 2 (Tabela 4), provavelmente devido ao acúmulo de sobras de ração e de metabólitos no ponto 1 (onde localizam-se os tanquesredes) os quais devido a movimentação da água foram carreados para o Ponto 2.

Tabela 4. Concentração de nitrogênio total na água, em mg. $\mathrm{L}^{-1}$, nos pontos de amostragem da piscicultura no Córrego Ribeirão Ponte Pensa, Santa Fé do Sul -SP, no período de março de 2008 à fevereiro de 2009

\begin{tabular}{cccccccccccc}
\hline & \multicolumn{10}{c}{ Meses } \\
\cline { 2 - 12 } $\begin{array}{c}\text { Pontos } \\
\text { amostrais }\end{array}$ & Mar & Abr & Mai & Jun & Jul & Ago & Set & Out & Nov & Jan & Fev \\
\hline P1 & 0,6 & 0,8 & 0,1 & 1,2 & 0,8 & 0,8 & 0,1 & 0,6 & 1,4 & 0,2 & 1,3 \\
P2 & 0,7 & 0,7 & 0,4 & 1,3 & 2,7 & 0,7 & 1,2 & 1,8 & 3,6 & 1,2 & 2,2 \\
P3 & 0,8 & 0,7 & 0,5 & 1,5 & 0,4 & 0,4 & 0,7 & 1,7 & 0,6 & 1,1 & 1,9 \\
\hline
\end{tabular}


Os padrões de qualidade segundo a Resolução CONAMA 357/05 para águas doces de classe 2, quando o nitrogênio for fator limitante para eutrofização, nas condições estabelecidas pelo órgão ambiental competente (valor de nitrogênio total após oxidação) não deverá ultrapassar 2,18 mg. $\mathrm{L}^{-1}$ para ambientes lóticos. Nota-se que nos Pontos 1 e 3 todos os valores registrados enquadram-se na legislação enquanto que no ponto 2 este valor ultrapassou o permitido em três amostras, sendo que a concentração máxima registrada foi $3,6 \mathrm{mg} \cdot \mathrm{L}^{-1}$ no mês de novembro de 2008. Próximo ao Ponto 2 também existem construções residenciais que de alguma forma podem estar contribuindo para os valores apresentados.

Nos três pontos amostrais da piscicultura localizada no Ribeirão da Ponte PensaSP a concentração de fósforo total durante os meses avaliados não ultrapassou o padrão para ambientes lóticos (0,1 mg. $\left.\mathrm{L}^{-1}\right)$ estabelecido pelo CONAMA 357/05.

As maiores concentrações de fósforo total foram registradas no Ponto 1 (Tabela 5) para todos os meses, com exceção do mês de fevereiro. Estas concentrações maiores em relação aos demais pontos amostrais podem ter ocorrido porque na área de cultivo há grande aporte de ração diariamente e o nutriente contido no alimento não é totalmente assimilado pelos peixes, além de que uma parte deste retorna ao ambiente através da excreção. A baixa renovação de água na área do cultivo contribui também com a sedimentação e acréscimo nos níveis de fósforo total no local, o que pode contribuir com a eutrofização do meio em longo prazo.

Todas as concentrações deste nutriente encontradas nos pontos amostrais são inferiores a $0,05 \mathrm{mg} \cdot \mathrm{L}^{-1}$ que segundo Boyd e Tucker (1998), são consideradas ideais para pisciculturas com sistema de tanques-rede.

Tabela 5. Concentração de fósforo total, em mg. $\mathrm{L}^{-1}$, nos pontos de amostragem da piscicultura no Córrego Ribeirão Ponte Pensa, Santa Fé do Sul - SP, no período de março de 2008 à fevereiro de 2009 


\section{Meses}

\begin{tabular}{|c|c|c|c|c|c|c|c|c|c|c|c|}
\hline \multirow{2}{*}{$\begin{array}{c}\text { Pontos } \\
\text { amostrais }\end{array}$} & \multicolumn{9}{|c|}{2008} & \multicolumn{2}{|c|}{2009} \\
\hline & Mar & Abr & Mai & Jun & Jul & Ago & Set & Out & Nov & Jan & Fev \\
\hline$r 1$ & 0,017 & 0,019 & 0,021 & $0,0<0$ & ודוס & $0<<$ & 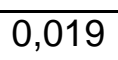 & $\longdiv { 1 4 }$ & 18 & 021 & I \\
\hline P2 & 0,012 & 0,008 & 0,009 & 0,007 & 0,004 & 0,008 & 0,00 & 0,007 & 0,011 & 0,011 & 0,020 \\
\hline P3 & 0,013 & 0,012 & 0,013 & 0,015 & 0,008 & 0,015 & 0,014 & 0,011 & 0,018 & 0,018 & 0,016 \\
\hline
\end{tabular}

\section{CONCLUSÃO}

$\mathrm{Na}$ piscicultura com sistema de cultivo em tanques-rede do Ribeirão da Ponte Pensa, município de Santa Fé do Sul - SP a temperatura da água manteve-se pouco variável nos três pontos de amostragem com oscilação de 23 a $30^{\circ} \mathrm{C}$ ao longo dos onze meses. Todos os valores de $\mathrm{pH}$ registrados nos três pontos amostrais enquadram-se no padrão estabelecido pelo CONAMA 357/05 para corpos d'água de classe 2 .

Em relação à concentração de oxigênio dissolvido, todas as amostras dos três pontos de coleta apresentaram concentrações superiores a $5 \mathrm{mg} \cdot \mathrm{L}^{-1}$ o que condiz com o padrão da legislação. No caso do nitrogênio total, observou-se que todas as concentrações encontradas nos pontos 1 e 3 enquadram-se no padrão do CONAMA 357/05, no entanto no ponto 2 a concentração deste nutriente foi superior ao permitido em três amostras. No que diz respeito ao fósforo total, todas as concentrações registradas durante os meses do estudo estiveram abaixo do padrão para ambientes lóticos estabelecido pelo CONAMA 357/05.

Os resultados deste estudo sugerem que a produção em tanques-rede no Ribeirão da Ponte Pensa - SP é uma atividade viável, desde que sejam tomadas certas precauções para evitar a degradação do ambiente aquático. Entre essas medidas está o monitoramento constante da qualidade da água e a otimização no uso da ração para que a mesma não seja desperdiçada, contribuindo para processos de eutrofização, buscando sempre respeitar a capacidade de suporte do ambiente aquático. 


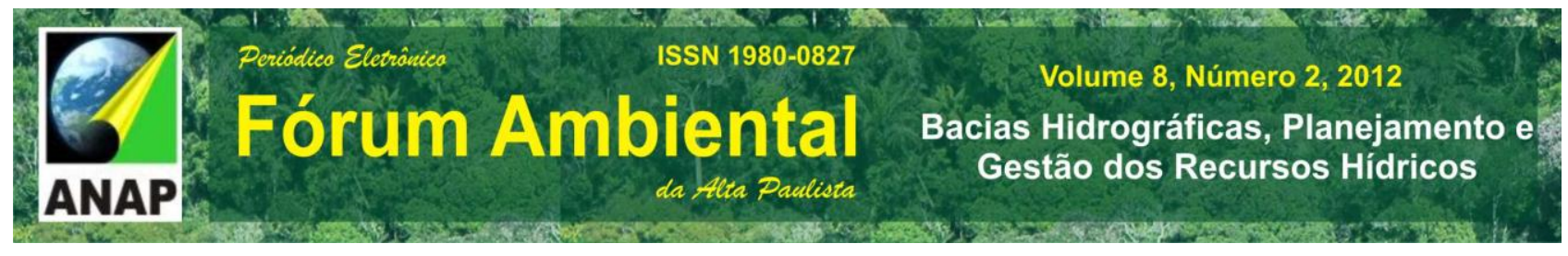

\section{REFERÊNCIAS}

APHA - AMERICAN PUBLIC HEALTH ASSOCIATION. - AWWA. AMERICAN WATER WORKS ASSOCIATION - WATER ENVIRONMENTAL FEDERATION - WPCF Standard methods for the examination of water and wastewater. $20^{\text {th }}$. Washington: APHA, 1998.

BACCARIN, A. E.; CAMARGO, A. F. M. Characterization and evaluation of the impact of feed management on the effluents of Nile Tilapia (Oreochromis niloticus). Culture Brazilian Archives of Biology and Technology, Curitiba, v. 48, n.1, p. 81-90, jan. 2005.

BOYD, C. E.; TUCKER, C. S. Pond aquaculture water quality management. Massachussets: Kluwer Academic Publishers, 1998. 700p.

BOYD, C. E.; SCHIMITTOU, H. R. Achievement of sustainable aquaculture through environmental management. Aquaculture Economics \& Management, Philadelphia, v.3, n.1. p.59-69, 1999.

BOYD, C. E. Guidelines for aquaculture effluent management at the farm-level. Aquaculture, Amsterdam, v.226. p.101-112. 2003.

COMITÊ DA BACIA HIDROGRÁFICA DO RIO SÃO JOSÉ DOS DOURADOS - CBHSJD. Disponível em:< http://www.comitesjd.sp.gov.br/apresentacao.htm> Acesso: 16 jan. 2009.

CONSELHO NACIONAL DO MEIO AMBIENTE - CONAMA. Resolucao $\mathbf{n}^{\circ} \mathbf{3 5 7}$, de 17 de março de 2005. Disponivel em: <http:www.mma.gov.br/port/conama> Acesso: 15 dez. 2008.

FOLKE, C.; KAUTSKY, N. Aquaculture with its Environment; Prospects for Sustainability. Ocean and Coastal Management, Orlando, v.17, p. 5-24, 1992.

GUO, L.; LI, Z. Effects of nitrogen and phosphorus from fish cage-culture on the communities of a shallow lake in middle Yangtze River basin of China. Aquaculture, Amsterdam, v.226, p.201-212, 2003. 
INSTITUTO BRASILEIRO DE GEOGRAFIA E ESTATISTICA- IBGE. Cidades@: Tres Lagoas MS. [S.I.: sn.], 2009. Disponivel em:

<http://www.ibge.gov.br/cidadesat/default.php>. Acesso: 19 dez. 2008.

KUBITZA, F. Qualidade da água na produção de peixes. Revista Panorama da Aquicultura, Rio de Janeiro, v. 8, n.46, p. 35-41,1998.

KUBITZA, F. 2000. Tilápia: tecnologia e planejamento na produção comercial. Jundiaí: F. KUBITZA, 2000. 289p.

QUEIROZ, J. F. A Relação do Uso de Rações com o Ambiente. Alimentação Animal, São Paulo, v.3, n.10, p. 108-111, 1998.

ROSS, B.; ROSS, L. G. The oxigen requeriments of Oreochromis niloticus under adverse conditions. In: FISHELSON, L.; YARON, Z. (eds.) International symposium on tilapia in aquaculture.Tel Aviv University, Israel 1983. p.134-143.

VALENTI, W. C. Aquaculture for sustainable development. In: VALENTI, W. C.; POLI, C. R; PEREIRA, J.A.; BORGHETTI, J.R. (Ed.) Aquicultura no Brasil, bases para um desenvolvimento sustentável. Brasilia: CNPQ/Ministerio da Ciencia e Tecnologia, 2000. p.17-24. 